\title{
Molecular differences between mature and immature dental pulp cells: Bioinformatics and preliminary results
}

\author{
LONG CHEN $^{1}$, YIFENG JIANG ${ }^{2}$ and ZHEN DU ${ }^{1}$ \\ ${ }^{1}$ Department of Stomatology, Linyi People's Hospital, Linyi, Shandong 276000; ${ }^{2}$ Stomatology Department of \\ Shandong Medical College, Linyi, Shandong 276002, P.R. China
}

Received August 9, 2017; Accepted January 5, 2018

DOI: $10.3892 / \mathrm{etm} .2018 .5847$

\begin{abstract}
Although previous studies have demonstrated that dental pulp stem cells (DPSCs) from mature and immature teeth exhibit potential for multi-directional differentiation, the molecular and biological difference between the DPSCs from mature and immature permanent teeth has not been fully investigated. In the present study, 500 differentially expressed genes from dental pulp cells (DPCs) in mature and immature permanent teeth were obtained from the Gene Expression Omnibus online database. Based on bioinformatics analysis using the Database for Annotation, Visualization and Integrated Discovery, these genes were divided into a number of subgroups associated with immunity, inflammation and cell signaling. The results of the present study suggest that immune features, response to infection and cell signaling may be different in DPCs from mature and immature permanent teeth; furthermore, DPCs from immature permanent teeth may be more suitable for use in tissue engineering or stem cell therapy. The Online Mendelian Inheritance in Man database stated that Sonic Hedgehog (SHH), a differentially expressed gene in DPCs from mature and immature permanent teeth, serves a crucial role in the development of craniofacial tissues, including teeth, which further confirmed that SHH may cause DPCs from mature and immature permanent teeth to exhibit different biological characteristics. The Search Tool for the Retrieval of Interacting Genes/Proteins database revealed that $\mathrm{SHH}$ has functional protein associations with a number of other proteins, including Glioma-associated oncogene (GLI)1, GLI2, growth arrest-specific protein 1, bone morphogenetic protein (BMP)2 and BMP4, in mice and humans. It was also demonstrated that SHH may interact with other genes to regulate the biological characteristics of DPCs. The results of the present study may provide a useful reference basis for
\end{abstract}

Correspondence to: Dr Zhen Du, Department of Stomatology, Linyi People's Hospital, 27 East Jiefang Road, Linyi, Shandong 276000, P.R. China

E-mail: duzhen0539@163.com

Key words: molecular difference, dental pulp cells, mature, immature, permanent teeth selecting suitable DPSCs and molecules for the treatment of these cells to optimize features for tissue engineering or stem cell therapy. Quantitative polymerase chain reaction should be performed to confirm the differential expression of these genes prior to the beginning of a functional study.

\section{Introduction}

Dental pulp cells (DPCs) include unique mesenchymal stem cells called dental pulp stem cells (DPSCs). Previous studies have demonstrated that DPSCs have the potential for multi-directional differentiation into osteoblasts, odontoblasts, adipocytes, chondrocytes and neural-like cells in vitro $(1,2)$ and may be useful seed cells for tissue engineering or stem cell therapy in a number of disease conditions (3-5). DPSCs may be obtained easily from deciduous or permanent teeth that are discarded as medical waste. Previous studies have compared the difference between DPSCs from deciduous teeth and permanent teeth (6); however, differences between DPSCs from permanent teeth of immature and mature patients have not yet been described. A previous study established a number of DPC lines isolated from extracted wisdom teeth as a cell source for induced pluripotent stem (iPS) cells and the donor teeth of these DPCs lines were divided into three developmental stages: Crown completed (CC), root forming (RF) and root completed (RC) (7). Teeth from 14-16 year old patients were defined as immature teeth (CC and RF), whereas teeth from 23-60 year old patients were defined as mature teeth (RC). The DPCs obtained from immature permanent teeth exhibited a much higher reprogramming potential compared with DPCs from mature permanent teeth (7). Furthermore, distal-less homeobox 4, a transcription factor that is highly expressed in DPCs from immature permanent teeth, significantly promoted human iPS cell generation as well as octamer-binding protein 3/4, sex-determining region $\mathrm{Y}$ box 2 and Kruppel-like factor 4 (7). These findings suggest that the biological characteristics of DPSCs differ in immature and mature permanent teeth. In the present study, the molecular characteristics of DPCs from immature and mature permanent teeth were compared based on bioinformatics analysis. The results of the present study may offer a basis for selecting a suitable preservation time for DPCs, as well as developing a suitable molecular intervention strategy for these cells to optimize features for tissue engineering or stem cell therapy. 


\section{Materials and methods}

Use of the Gene Expression Omnibus (GEO) database to obtain differentially expressed genes in DPCs from mature and immature permanent teeth. Tamaoki et al (7) previously used an array to analyze gene expression profiles and investigate the effect of the developmental stage of teeth on global gene expression in human DPCs. The microarray data were deposited in the GEO online database (https://www.ncbi.nlm. nih.gov/geo/query/acc.cgi?acc=GSE52853), the detailed information for which was described in a previous article and the online webpage (https://www.ncbi.nlm.nih.gov/geo/query/acc. cgi?acc=GSE52853) (7). In the present study, differentially expressed genes in DPCs from mature and immature permanent teeth were acquired from the GEO database. The protocol was described as follows: i) Search 'dental pulp cells' in the GEO database (http://www.ncbi.nlm.nih.gov/gds/) to obtain the GSE52853 datasets (Title: Human Dental Pulp Cells Mature vs. Immature stages); ii) GEO2R analysis: Use GEO2R, one of GEO's own data analysis tools, to identify differentially expressed genes in DPCs from mature and immature permanent teeth. The default GEO2R statistical tests were applied. The expression value was stated with reference to that of mature permanent teeth and a moderated t-statistic was used to compare the expression of genes in DPCs from mature permanent teeth with those from immature permanent teeth. Genes that differed most significantly $(\mathrm{P}<0.001 ; \mid \log \mathrm{FCl}>1)$ were identified and were selected for further analysis (8). Some genes with $\mathrm{P}<0.001$ and $\log \mathrm{FCl}<1$ that serve a crucial role in tooth development were also included in the present study. In total, 500 differentially expressed genes were selected for further analysis.

Use of the Database for Annotation, Visualization and Integrated Discovery (DAVID) to evaluate 500 differentially expressed genes in DPCs from mature and immature permanent teeth. The DAVID bioinformatics database is an integrated online database that includes a number of powerful annotation resources, including Gene Ontology (GO), Biocarta and Kyoto Encyclopedia of Genes and Genomes (KEGG) databases (9). In the present study, the DAVID bioinformatics database was used to analyze the protein associations, biological characteristics and functional characteristics of differentially expressed genes in DPCs from mature and immature permanent teeth. The protocol was as follows: i) Access DAVID (https://david. ncifcrf.gov/) and copy the 500 differentially expressed gene names into box A; ii) select the 'official gene symbol' as the gene identifier type and click the 'Submit List' button; iii) choose 'Homo sapiens' as annotations; and iv) select 'Start Analysis'. The modified Fisher's Exact test was used in the present study, and $\mathrm{P}<0.05$ was considered to indicate a statistically significant difference.

Use of online mendelian inheritance in man (OMIM) to analyze the genetic characteristics of sonic hedgehog (SHH). The OMIM database is a comprehensive, authoritative compendium of human genes and genetic phenotypes that is freely available online. OMIM focuses on the association between genotype and phenotype and the entries contain links to other genetics resources. SHH was differentially expressed in DPCs from mature and immature permanent teeth and exhibited complex effects in DPCs. In the present study, the OMIM database was used to analyze the genetic characteristics of SHH. The protocol was as follows: i) Access the OMIM website (http://www.omim. org/), and enter 'SHH' in the search box; ii) select ' 600725 . SONIC HEDGEHOG; SHH'; iii) select 'MGI Mouse Gene' from the 'Animal Models' menu; and iv) review the genetic information about $\mathrm{SHH}$, including 'GO Classifications', 'Mutations, Alleles, and Phenotypes' and 'Expression'.

Use of the search Tool for the Retrieval of Interacting Genes/Proteins (STRING) online database to analyze functional protein association networks of SHH. STRING is a database of known and predicted protein-protein interactions that includes direct (physical) and indirect (functional) associations stemming from computational prediction, interactions aggregated from other primary databases and information transfer between organisms (10). In the present study, the STRING online database was used to analyze functional protein association networks of SHH. The protocol was as follows: i) Access the STRING online database (http:// www.string-db.org/); ii) enter the protein name 'SHH' and choose organism as 'Homo sapiens' or 'Mus musculus' and search; and iii) obtain the network chart of functional protein association networks of SHH.

\section{Results}

A total of 500 genes in DPCs were demonstrated to be differentially expressed in mature and immature permanent teeth. A number of differentially expressed genes in were identified in DPCs from mature and immature permanent teeth via analysis of GEO2R in the GEO database. The 500 genes that differed most significantly $(\mathrm{P}<0.001)$ were selected for further analysis using the DAVID online database.

Differentially expressed genes in DPCs from mature and immature permanent teeth may be divided into immunity, inflammation and cell signaling-associated biological process subgroups. Following DAVID analysis, the 500 differentially expressed genes in DPCs were divided into subgroups based on the GO term 'biological process'. The majority of these subgroups were separated into three groups, associated with immunity, inflammation and cell signaling. Genes in the immune-associated groups (Table I) were demonstrated to regulate lymphocyte activation and proliferation, antigen processing and negative regulation of immune system processes. A total of two genes, including cluster of differentiation 24 and SHH, existed in almost all subgroups. The 500 differentially expressed genes were enriched into different KEGG pathway subgroups based on DAVID analysis. A total of four genes, including $\gamma$-interferon-inducible lysosomal thiol reductase, human leukocyte antigen class II histocompatibility antigen (HLA)-DR $\beta 5$ chain, HLA DP $\beta 1$ chain and leukotriene A-4 hydrolase, were enriched into the 'Antigen processing and presentation' pathway (Fig. 1). Genes in the inflammation-associated groups (Table II) may act to regulate leukocyte migration, proliferation, activation and chemotaxis, mononuclear cell proliferation and the defense response. Genes in the cell signaling-associated groups (Table III) 
Table I. Differentially expressed genes in dental pulp cells from mature and immature permanent teeth were enriched into immunity associated subgroups based on 'Biological Process' terms analyzed using the Database for Annotation, Visualization and Integrated Discovery.

Differentially expressed genes

Term Upregulated in IPT

Downregulated in IPT

Fold enrichment

Regulation of lymphocyte activation

Regulation of lymphocyte proliferation

Regulation of B cell activation

Negative regulation of lymphocyte activation

Regulation of B cell proliferation

Antigen processing and presentation of peptide or polysaccharide antigen via MHC class II Negative regulation of immune system process
CD24, LAG3

CD24

CD24

CD24, LAG3

$\mathrm{CD} 24$

HLA-DPB1

CD24, LAG3
INHBA, NCK2, CDKN1A, TNFRSF4, SHH

NCK2, CDKN1A, TNFRSF4, SHH

3.677229 INHBA, CDKN1A, TNFRSF4

4.683562

6.097814

INHBA, SHH

5.759046

CDKN1A, TNFRSF4

8.330049

7.067921

3.746849

IPT, immature permanent teeth.

Antigen processing and presentation

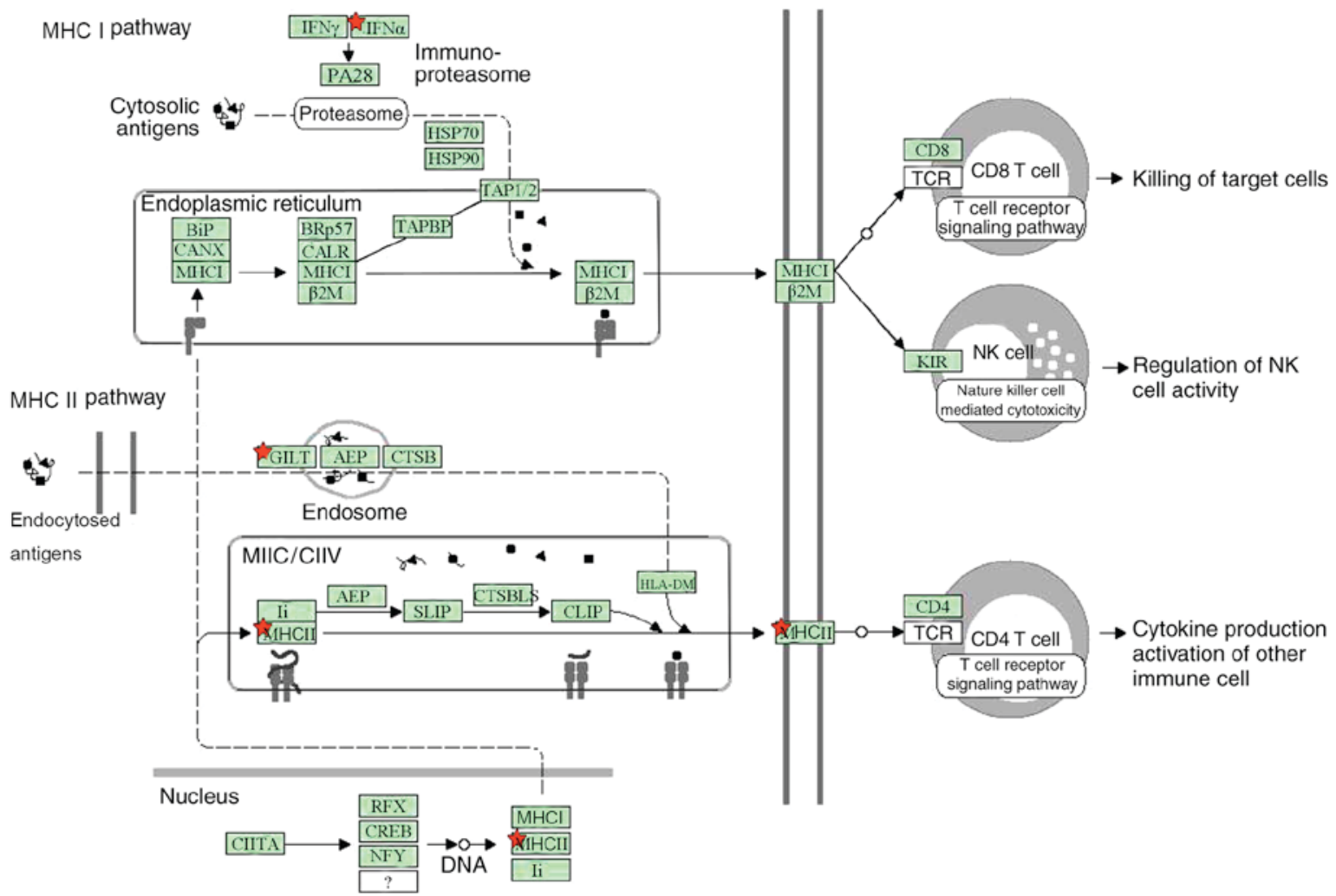

Figure 1. Differentially expressed genes (red stars) in dental pulp cells from mature and immature permanent teeth are enriched into an 'antigen process and presentation pathway' based on the Kyoto Encyclopedia of Genes and Genomes and analyzed by the Database for Annotation, Visualization and Integrated Discovery.

regulate chemotaxis, cell-cell signaling transduction, cell surface receptor linked signal transduction and G-protein coupled receptor protein signaling pathways. These findings suggest that immune features, response to infection and signaling may be different in DPCs from mature and immature permanent teeth.
Genetic characteristics of SHH. The OMIM database analysis revealed that $\mathrm{SHH}$ is expressed in cellular projections, extracellular regions and plasma membranes and is associated with biological processes, including cell proliferation, cell differentiation and immune system processes. It was also demonstrated that, in the craniofacial region, 
Table II. Differentially expressed genes in DPCs from mature and immature permanent teeth were enriched into inflammation-associated subgroups based on 'Biological Process' terms analyzed using the Database for Annotation, Visualization and Integrated Discovery.

\begin{tabular}{|c|c|c|c|}
\hline \multirow[b]{2}{*}{ Term } & \multicolumn{2}{|c|}{ Differentially expressed genes } & \multirow[b]{2}{*}{ Fold enrichment } \\
\hline & Upregulated in IPT & Downregulated in IPT & \\
\hline Leukocyte migration & $\mathrm{CD} 24$ & AZU1, PODXL, ITGAM & 6.819923 \\
\hline Regulation of leukocyte activation & CD24, LAG3 & $\begin{array}{l}\text { INHBA, TNFRSF4, SHH, NCK2, } \\
\text { CDKN1A }\end{array}$ & 3.278493 \\
\hline Regulation of mononuclear cell proliferation & $\mathrm{CD} 24$ & NCK2, CDKN1A, TNFRSF4, SHH & 4.627805 \\
\hline Regulation of leukocyte proliferation & $\mathrm{CD} 24$ & NCK2, CDKN1A, TNFRSF4, SHH & 4.627805 \\
\hline Positive regulation of leukocyte proliferation & $\mathrm{CD} 24$ & NCK2, CDKN1A, TNFRSF4 & 5.553366 \\
\hline Negative regulation of leukocyte activation & CD24, LAG3 & INHBA, SHH, & 5.455939 \\
\hline Leukocyte chemotaxis & & AZU1, ITGAM & 6.303821 \\
\hline Defense response & $\begin{array}{l}\text { F11R, TRPV1, } \\
\text { CD24, AOC3 }\end{array}$ & $\begin{array}{l}\text { C3AR1, DEFB4A, TNFRSF4, AZU1, } \\
\text { INHBA, APOL1 }\end{array}$ & 1.643435 \\
\hline
\end{tabular}

IPT, immature permanent teeth.

Table III. Differentially expressed genes in dental pulp cells from mature and immature permanent teeth were enriched into cell signaling-associated subgroups based on 'Biological Process' terms analyzed by the Database for Annotation, Visualization and Integrated Discovery.

\begin{tabular}{|c|c|c|c|}
\hline \multirow[b]{2}{*}{ Term } & \multicolumn{2}{|c|}{ Differentially expressed genes } & \multirow[b]{2}{*}{ Fold enrichment } \\
\hline & Upregulated in IPT & Downregulated in IPT & \\
\hline Cell-cell signaling & $\mathrm{CD} 24$ & $\begin{array}{l}\text { WNT5A, GRM5, SHH, GRM4, INHBA, } \\
\text { NMUR2, SLC1A1, CLN8, LTA, CACNA1B }\end{array}$ & 2.202835 \\
\hline Taxis & & AZU1, C3AR1, DEFB4A, ITGAM & 3.887356 \\
\hline Chemotaxis & & $\begin{array}{l}\text { AZU1, C3AR1, CMKLR1, DEFB4A, } \\
\text { ITGAM, SLIT2 }\end{array}$ & 3.887356 \\
\hline $\begin{array}{l}\text { Cell surface recept or linked } \\
\text { signal transduction }\end{array}$ & $\begin{array}{l}\text { GLI1, ADAM32, KLRF1, } \\
\text { CD24, AXIN2, LAG3 }\end{array}$ & $\begin{array}{l}\text { WNT5A, C3AR1, OR5H1, TAS2R5, TACR2, } \\
\text { OR11A1, DEFB4A, OR8U1, SHH, ITGAM, } \\
\text { AZU1, NMUR2, CMKLR1, GPR78, KCNK2, } \\
\text { GRM5, OR6M1, INHBA, NCK2, GPR32, } \\
\text { GRM4, OR52K2, KNG1 }\end{array}$ & 1.549916 \\
\hline $\begin{array}{l}\text { G-protein coupled receptor } \\
\text { protein signaling pathway }\end{array}$ & GPRC5C & $\begin{array}{l}\text { KNG1, C3AR1, OR5H1, NXPH4, TAS2R5, } \\
\text { CMKLR1, TACR2, GPR78, OR11A1, NPR3, } \\
\text { DEFB4A, OR8U1, KCNK2, OR6M1, AZU1, } \\
\text { GRM5, GRM4, GPR32, PTGIR, OR52K2, } \\
\text { NMUR2, OR1F2P }\end{array}$ & 1.592328 \\
\hline Metal ion transport & TRPV1, KCNK12 & $\begin{array}{l}\text { NMUR2, KCNK2, CALHM1, SLC17A3, } \\
\text { SLC17A4, CAMK2D, SLC39A5, KCNQ2, } \\
\text { CACNA1B }\end{array}$ & 2.006377 \\
\hline
\end{tabular}

IPT, immature permanent teeth.

SHH mutation could lead to a number of deformities in mice (Table IV) or humans (Table V), including abnormal maxillary morphology, abnormal incisor morphology, tooth development arrest and single median maxillary incisor. These findings further confirm that SHH serves a crucial role in the development of teeth and may also cause DPCs from mature and immature permanent teeth to exhibit different biological characteristics.

Functional protein association networks of SHH. Based on STRING analysis, it was demonstrated that SHH has functional protein associations with a number of other proteins 
Table IV. Craniofacial phenotypes in an SHH mutant mouse model.

\begin{tabular}{|c|c|}
\hline Mouse genotype & Mouse phenotypes \\
\hline $\mathrm{Shh}^{\mathrm{Dsh}} / \mathrm{Shh}^{\mathrm{Dsh}}$ & AM \\
\hline $\mathrm{Shh}^{\mathrm{tm} 1 \mathrm{Chg}} / \mathrm{Shh}^{\mathrm{tm} 1 \mathrm{Chg}}$ & ACM, ACBM, ANSM \\
\hline $\mathrm{Shh}^{\mathrm{tm} 3(\mathrm{Cre}) \mathrm{Chg}} / \mathrm{Shh}^{\mathrm{tm} 3(\mathrm{Cre}) \mathrm{Chg}}$ & $\mathrm{ACM}$ \\
\hline $\mathrm{Shh}^{\mathrm{Dsh}} / \mathrm{Shh}^{+}$ & ACMM, ANM, AFM, AIM, OBM, AMM \\
\hline $\mathrm{Shh}^{\mathrm{Hx}} / \mathrm{Shh}^{+}$ & $\mathrm{CHP}$ \\
\hline \multicolumn{2}{|l|}{$\mathrm{Shh}^{\mathrm{tm} 1 \mathrm{Amc}} / \mathrm{Shh}^{\mathrm{tm} 2 \mathrm{Amc}}$} \\
\hline $\operatorname{Tg}(\mathrm{KRT} 14-\mathrm{Cre}) 1 \mathrm{Amc} / 0$ (conditional) & $\begin{array}{l}\text { ACMM, AAP, AICM, AMCM, SM, AABM, ATD, GRI, } \\
\text { GRM, ADM, AEM, ANBM, APSF, PSH, CSP }\end{array}$ \\
\hline \multicolumn{2}{|l|}{$\mathrm{Shh}^{\mathrm{tm} 1 \mathrm{Amc}} / \mathrm{Shh}^{\mathrm{tm} 2 \mathrm{Amc}}$} \\
\hline Tg(Thy1-Cre)703Vaw/? (conditional) & $\mathrm{ACM}$ \\
\hline \multicolumn{2}{|l|}{ 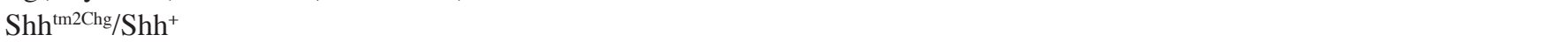 } \\
\hline $\operatorname{Tg}($ Sox2-Cre) 1 Amc/0 (conditional) & ANM, AMSM, PSFMM, CSP \\
\hline \multicolumn{2}{|c|}{$\begin{array}{l}\text { ACM, abnormal craniofacial morphology; ACBM, abnormal craniofacial bone morphology; ACMM, abnormal cranium morphology; ANM, } \\
\text { abnormal neurocranium morphology; AFM, abnormal frontal bone morphology; AIM, abnormal intraparietal bone morphology; OBM, occip- } \\
\text { ital bone foramen; AAP, absent alveolar process; AMM, abnormal maxilla morphology; AMSM, abnormal maxillary shelf morphology; AICM, } \\
\text { abnormal incisor morphology; AMCM, abnormal molar crown morphology; SM, small molars; AABM, abnormal ameloblast morphology; } \\
\text { ATD, arrest of teeth development; GRI, growth retardation of incisor; GRM, growth retardation of morlar; ADM, abnormal dentin morphology; } \\
\text { AEM, abnormal enamel morphology; ANBM, abnormal nasal bone morphology; ANSM, abnormal nasal spit morphology; APSM, abnormal } \\
\text { palate shelf fusion; PSFMM, palate shelf fail to meet at midline; PSH, palate shelf hypoplasia; CHP, cleft hard plate; CSP, cleft secondary } \\
\text { palate; AM, absent mouth; SHH, Sonic hedgehog. }\end{array}$} \\
\hline
\end{tabular}

Table V. Craniofacial phenotypes in patients with $\mathrm{SHH}$-associated diseases.

Diseases associated with $\mathrm{SHH}$

Holoprosencephaly 3

Microphthalmia, isolated, with coloboma 5

Solitary median maxillary central incisor
Human phenotypes

MF, malar flattening; MR, midface retrusion; AN, abnormality of the nose; PB, proboscis; CC, cyclopia; HL, hypotelorism; OC, oral cleft; AP, anophthalmia; MP, microphthalmia; BMP, bilateral microphthalmia; IFC, irido-fundal coloboma; SP, short philtrum; TULV, tented upper lip vermilion; CUL, cleft upper lip; CP, cleft palate; TP, torus palatinus; PMPP, prominent median palate palate; SMMI, single median maxillary incisor; ARN, anteverted nares; SN, short nose; CA, choanal atresia; NNB, narrow nasal bridge; MS, midnasal stenosis; ABN, abnormality of the nasopharynx; AS, anosmia; CB, coloboma; ICB, iris coloboma; MP, microcephaly; SHH, Sonic hedgehog.

in mice and humans, including glioma-associated oncogene (GLI)1, GLI2, growth arrest-specific protein 1 (GAS1), bone morphogenetic protein (BMP)2 and BMP4 (Fig. 2). These findings indicate that $\mathrm{SHH}$ may interact with other genes to regulate the biological characteristics of DPCs.

\section{Discussion}

Dental pulp tissues may be derived from migrating cranial neural crest cells during development; these tissues harbor various populations of multipotential stem cells and are useful cell resource for dental tissues, bone and neuron regeneration $(1,11,12)$. DPSCs may be obtained from deciduous, inflamed deciduous, immature permanent or mature permanent teeth and the cells from each source may exhibit different biological characteristics (13). A previous study demonstrated that inflamed human dental pulp retains tissue regeneration potential (14). However, it has been suggested that inflamed DPSCs have reduced stemness and immunomodulatory properties and the expression of a number of molecules is different compared with non-inflamed DPSCs (15). This implies that inflamed DPSCs may not be a viable therapeutic cell source in cases of graft versus host disease and organ rejection (15). Previous findings have also demonstrated that DPSCs from deciduous teeth exhibit an upregulation in genes that participate in pathways associated with cell proliferation and the extracellular matrix including, fibroblast growth factor and tumor growth factor $\beta$ (16-18). Although it has previously been reported that DPSCs from mature and immature teeth have the potential for multi-directional differentiation, the molecular and biological differences between DPSCs from mature and immature permanent teeth has not been fully 

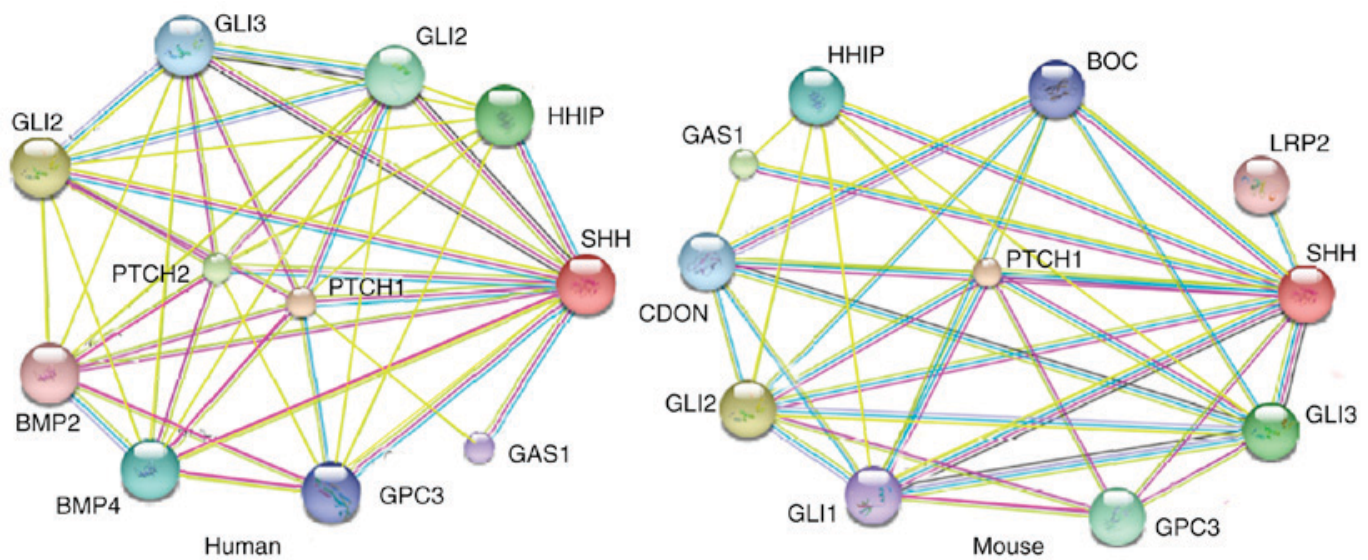

Line legends:
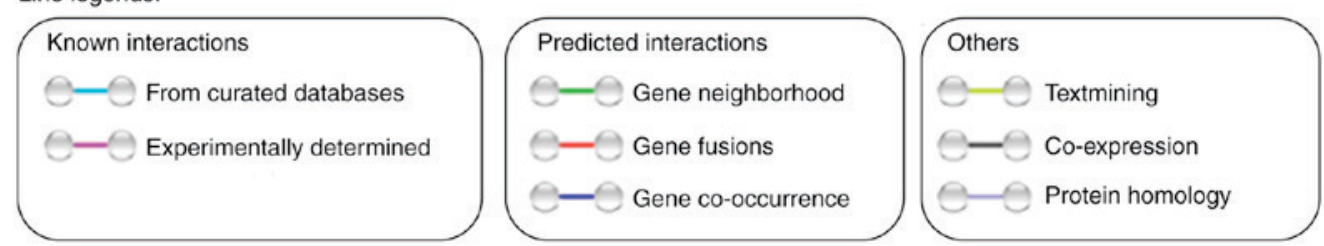

Figure 2. Functional protein association networks of SHH analyzed using Search Tool for the Retrieval of Interacting Genes/Proteins. SHH, sonic hedgehog.

investigated. The results of the present study demonstrate that a number of differentially expressed genes in DPCs from mature and immature permanent teeth are associated with immunity, inflammation and cell signaling; furthermore, the findings herein suggest that immune features, response to infection and signaling may be different in DPCs from mature and immature permanent teeth. The differential expression patterns of genes also indicate that DPCs from immature permanent teeth may be more suitable for tissue engineering or stem cell therapy. In the present study, only one gene exhibited a statistically significant difference for the adjusted $P$ (adj.P) value (adj.P<0.05), suggesting that there may be some level of error. Therefore, the differentially expressed genes with a high $\mid \log \mathrm{FCl}$ value should be focused on, and quantitative polymerase chain reaction (qPCR) should be performed to confirm differential expression prior to starting a functional study.

SHH, a mammalian homologue of the Drosophila secreted morphogen hedgehog, serves crucial role in the development of many tissues (19). Previous studies have demonstrated that $\mathrm{SHH}$ is expressed and serves key roles during all stages of tooth development, including tooth initiation, patterning and eruption (20-22). SHH mutation results in retarded grown, teeth malformation and mispatterned teeth in mice. The results of the present study demonstrate that $\mathrm{SHH}$, which is differentially expressed in DPCs from mature and immature permanent teeth, is associated with many subgroups based on biological process GO terms. OMIM database analysis indicated that $\mathrm{SHH}$ serves a crucial role in the development of craniofacial tissues, suggesting that the differential expression of $\mathrm{SHH}$ may be responsible for the different biological characteristics of DPCs from mature and immature permanent teeth. Previous studies have also reported that $\mathrm{SHH}$ protein could bind to Patched to release Smoothened to activate the downstream signaling cascade (23). GLI1-3, Huntington-interacting protein and GAS1 serve crucial roles in regulating the SHH pathway (24-26). In the present study, STRING database analysis revealed that
SHH has functional protein associations with a number of other proteins, including GLI1, GLI2, GAS1, BMP2 and BMP4 in mice and humans. This suggests that $\mathrm{SHH}$ may interact with other genes to regulate the biological characteristics of DPCs.

In conclusion, 500 differentially expressed genes were identified in DPCs from mature and immature permanent teeth using the GEO online database. Based on bioinformatics analysis using the DAVID database, these genes were enriched into a number of subgroups associated with immune features, inflammation and cell signaling. These findings suggest that immune features, response to infection and signaling pathways may differ between DPCs from mature and immature permanent teeth and that DPCs from immature permanent teeth may be more suitable for tissue engineering or stem cell therapy. OMIM database analysis demonstrated that $\mathrm{SHH}$ serves crucial role in the development of craniofacial tissues, including teeth, and may be responsible for the biological differences in DPCs from mature and immature permanent teeth. The results of the present study may provide a basis for future functional experiments, including selecting suitable DPSCs for use in tissue engineering and stem cell therapy, as well as selecting intervention molecules to optimize the features of these tissues. qPCR may be required to confirm the differential expression prior to the launch of a functional study.

\section{Competing interests}

The authors declare that they have no competing interests.

\section{References}

1. Gronthos S, Brahim J, Li W, Fisher LW, Cherman N, Boyde A, DenBesten P, Robey PG and Shi S: Stem cell properties of human dental pulp stem cells. J Dent Res 81: 531-535, 2002.

2. Waddington RJ, Youde SJ, Lee CP and Sloan AJ: Isolation of distinct progenitor stem cell populations from dental pulp. Cells Tissues Organs 189: 268-274, 2009. 
3. Gandia C, Armiñan A, García-Verdugo JM, Lledó E, Ruiz A Miñana MD, Sanchez-Torrijos J, Payá R, Mirabet V, CarbonellUberos F, et al: Human dental pulp stem cells improve left ventricular function, induce angiogenesis, and reduce infarct size in rats with acute myocardial infarction. Stem Cells 26: 638-645, 2008.

4. Mead B, Logan A, Berry M, Leadbeater W and Scheven BA: Dental pulp stem cells, a paracrine-mediated therapy for the retina. Neural Regen Res 9: 577-578, 2014.

5. Nicola FC, Rodrigues LP, Crestani T, Quintiliano K, Sanches EF, Willborn S, Aristimunha D, Boisserand L, Pranke P and Netto CA: Human dental pulp stem cells transplantation combined with treadmill training in rats after traumatic spinal cord injury. Braz J Med Biol Res 49: e5319, 2016.

6. Majumdar D, Kanafi M, Bhonde R, Gupta P and Datta I: differential neuronal plasticity of dental pulp stem cells from exfoliated deciduous and permanent teeth towards dopaminergic neurons. J Cell Physiol 231: 2048-2063, 2016.

7. Tamaoki N, Takahashi K, Aoki H, Iida K, Kawaguchi T, Hatakeyama D, Inden M, Chosa N, Ishisaki A, Kunisada T, et al: The homeobox gene DLX4 promotes generation of human induced pluripotent stem cells. Sci Rep 4: 7283, 2014.

8. Wu S, Wu F and Jiang Z: Identification of hub genes, key miRNAs and potential molecular mechanisms of colorectal cancer. Oncol Rep 38: 2043-2050, 2017.

9. Huang da W, Sherman BT and Lempicki RA: Systematic and integrative analysis of large gene lists using DAVID bioinformatics resources. Nat Protoc 4: 44-57, 2009.

10. Szklarczyk D, Franceschini A, Wyder S, Forslund K, Heller D, Huerta-Cepas J, Simonovic M, Roth A, Santos A, Tsafou KP, et al: STRING v10: Protein-protein interaction networks, integrated over the tree of life. Nucleic Acids Res 43 (Database issue) D447-D452, 2015.

11. Chang CC, Chang KC, Tsai SJ, Chang HH and Lin CP: Neurogenic differentiation of dental pulp stem cells to neuron-like cells in dopaminergic and motor neuronal inductive media. J Formos Med Assoc 113: 956-965, 2014.

12. Bray AF, Cevallos RR, Gazarian K and Lamas M: Human dental pulp stem cells respond to cues from the rat retina and differentiate to express the retinal neuronal marker rhodopsin. Neuroscience 280: 142-155, 2014.

13. Rosa V, Dubey N, Islam I, Min KS and Nor JE: Pluripotency of stem cells from human exfoliated deciduous teeth for tissue engineering. Stem Cells Int 2016: 5957806, 2016.

14. Alongi DJ, Yamaza T, Song Y, Fouad AF, Romberg EE, Shi S, Tuan RS and Huang GT: Stem/progenitor cells from inflamed human dental pulp retain tissue regeneration potential. Regen Med 5: 617-631, 2010
15. Yazid FB, Gnanasegaran N, Kunasekaran W, Govindasamy V and Musa S: Comparison of immunodulatory properties of dental pulp stem cells derived from healthy and inflamed teeth. Clin Oral Investig 18: 2103-2112, 2014.

16. Nakamura S, Yamada Y, Katagiri W, Sugito T, Ito K and Ueda M Stem cell proliferation pathways comparison between human exfoliated deciduous teeth and dental pulp stem cells by gene expression profile from promising dental pulp. J of Endod 35: $1536-1542,2009$

17. Wang X, Sha XJ, Li GH, Yang FS, Ji K, Wen LY, Liu SY, Chen L, Ding Y and Xuan K: Comparative characterization of stem cells from human exfoliated deciduous teeth and dental pulp stem cells. Arch Oral Biol 57: 1231-1240, 2012.

18. Volponi AA, Pang Y and Sharpe PT: Stem cell-based biological tooth repair and regeneration. Trends Cell Biol 20: 715-722, 2010

19. Varjosalo M and Taipale J: Hedgehog: Functions and mechanisms. Genes Dev 22: 2454-2472, 2008.

20. Li J, Feng J, Liu Y, Ho TV, Grimes W, Ho HA, Park S, Wang S and Chai Y: BMP-SHH signaling network controls epithelial stem cell fate via regulation of its niche in the developing tooth. Dev Cell 33: 125-135, 2015.

21. Hu X, Zhang S, Chen G, Lin C, Huang Z, Chen Y and Zhang Y: Expression of SHH signaling molecules in the developing human primary dentition. BMC Dev Biol 13: 11, 2013.

22. Kumamoto H, Ohki K and Ooya K: Expression of Sonic hedgehog (SHH) signaling molecules in ameloblastomas. J Oral Pathol Med 33: 185-190, 2004.

23. Villavicencio EH, Walterhouse DO and Iannaccone PM: The sonic hedgehog-patched-gli pathway in human development and disease. Am J Hum Genet 67: 1047-1054, 2000.

24. Bai CB, Auerbach W, Lee JS, Stephen D and Joyner AL: Gli2, but not Gli1, is required for initial Shh signaling and ectopic activation of the Shh pathway. Development 129: 4753-4761, 2002.

25. Gan H, Liu H, Zhang H, Li Y, Xu X, Xu X and Xu J: SHh-Gli1 signaling pathway promotes cell survival by mediating baculoviral IAP repeat-containing 3 (BIRC3) gene in pancreatic cancer cells. Tumour Biol 37: 9943-9950, 2016.

26. Allen BL, Tenzen T and McMahon AP: The Hedgehog-binding proteins Gas1 and Cdo cooperate to positively regulate Shh signaling during mouse development. Genes Dev 21: 1244-1257, 2007.

This work is licensed under a Creative Commons

Attribution-NonCommercial-NoDerivatives 4.0 International (CC BY-NC-ND 4.0) License. 